\title{
Delayed evoked potentials in zebra finch (Taeniopygia guttata) under midazolam-butorphanol-isoflurane anesthesia
}

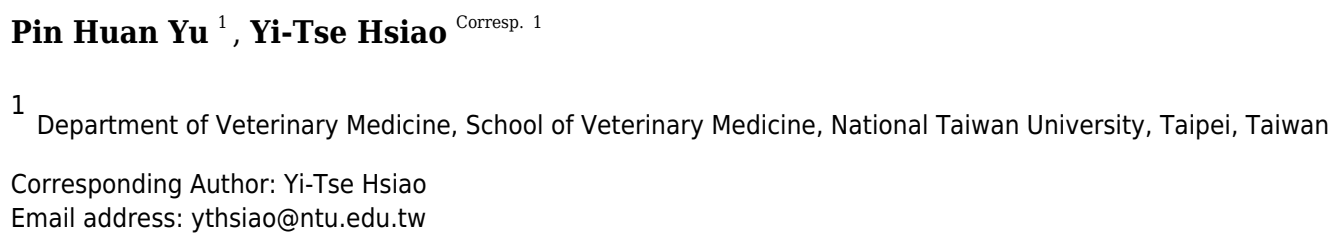

Avian animals are visually inclined, which has caused them to attract increasing attention for visual neurophysiology or electrophysiology studies, including the study of the visual evoked potential (VEP). VEP has developed into an investigative tool for understanding the physiology and the pathology of the visual pathway. Chemical restraint is a common method to minimize motion artifacts in animals when acquiring VEP data, but little is known about its influence on the signal in an avian animal. In addition, it is difficult to make comparisons between conscious state data when the animals are ultimately under anesthesia. Therefore, finding drugs and developing protocols that have an acceptable effect is valuable. We compared the local field potentials of physically and chemically restrained zebra finches (Taeniopygia guttata), a small avian species, to simulate a relatively challenging recording condition. Finches were sedated with midazolambutorphanol, and anesthesia was maintained by isoflurane. Electrodes were implanted into the left nucleus rotundus, which is a visual nucleus in birds. The VEPs of the control group $(\mathrm{N}=3)$ were recorded after they fully recovered and were restrained by towels. The other birds $(\mathrm{N}=3)$ were recorded under anesthesia. The results show that without the visual stimuli, anesthesia generally suppressed the overall power of field potentials. However, by focusing on the spectra during VEPs, visual stimuli still triggered significant VEPs in frequencies below $30.8 \mathrm{~Hz}$, which were even stronger than towel-restrained birds. The drugs also prolonged the latency of the VEP, increased the duration of the VEP when compared to towel-restrained birds. As regard to towel-restrained zebra finches, the field potentials were less synchronized and may need data preprocessing to have clear VEPs. In conclusion, the current study presents evidence of basic VEP for zebra finch under midazolam-butorphanol-isoflurane anesthesia with a protocol that is a safe and feasible anesthetic combination for chemical restraint, which is particularly useful for small animals when obtaining evoked potentials. 


\section{Delayed evoked potentials in zebra finch (Taeniopygia}

\section{2 guttata) under midazolam-butorphanol-isoflurane anesthesia}

3

4

5 Pin Huan $\mathrm{Yu}^{1}$, Yi-Tse Hsiao ${ }^{1}$

6

7 Department of Veterinary Medicine, School of Veterinary Medicine, National Taiwan

8 University, Taipei, Taiwan

9

10 Corresponding Author:

11 Yi-Tse Hsiao

12 No. 1, Sec. 4, Roosevelt Rd., Taipei 10617, Taiwan (R.O.C.)

13 Email address: ythsiao@ntu.edu.tw 


\section{Abstract}

16 Avian animals are visually inclined, which has caused them to attract increasing attention for

17 visual neurophysiology or electrophysiology studies, including the study of the visual evoked

18 potential (VEP). VEP has developed into an investigative tool for understanding the physiology

19 and the pathology of the visual pathway. Chemical restraint is a common method to minimize

20 motion artifacts in animals when acquiring VEP data, but little is known about its influence on

21 the signal in an avian animal. In addition, it is difficult to make comparisons between conscious

22 state data when the animals are ultimately under anesthesia. Therefore, finding drugs and

23 developing protocols that have an acceptable effect is valuable. We compared the local field

24 potentials of physically and chemically restrained zebra finches (Taeniopygia guttata), a small

25 avian species, to simulate a relatively challenging recording condition. Finches were sedated

26 with midazolam-butorphanol, and anesthesia was maintained by isoflurane. Electrodes were

27 implanted into the left nucleus rotundus, which is a visual nucleus in birds. The VEPs of the

28 control group $(\mathrm{N}=3)$ were recorded after they fully recovered and were restrained by towels.

29 The other birds $(\mathrm{N}=3)$ were recorded under anesthesia. The results show that without the visual

30 stimuli, anesthesia generally suppressed the overall power of field potentials. However, by 
31 focusing on the spectra during VEPs, visual stimuli still triggered significant VEPs in

32 frequencies below $30.8 \mathrm{~Hz}$, which were even stronger than towel-restrained birds. The drugs also

33 prolonged the latency of the VEP, increased the duration of the VEP when compared to towel-

34 restrained birds. As regard to towel-restrained zebra finches, the field potentials were less

35 synchronized and may need data preprocessing to have clear VEPs. In conclusion, the current

36 study presents evidence of basic VEP for zebra finch under midazolam-butorphanol-isoflurane

37 anesthesia with a protocol that is a safe and feasible anesthetic combination for chemical

38 restraint, which is particularly useful for small animals when obtaining evoked potentials. 


\section{Introduction}

40 Evoked potentials are voltage fluctuations of nervous systems which are triggered by the

41 occurrences of physical or mental events (Picton et al. 2000). Evoked potentials have been used

42 for intraoperative neurophysiologic monitoring and experimental purposes under anesthesia

43 (Bithal 2014; Cecchetto et al. 2015). Visual evoked potentials (VEPs) were identified as a

44 neurophysiologic test that could reflect the integrity of the visual pathways. Hence, VEPs came

45 to be used as a tool for intraoperative monitoring during major surgery (Sharika et al. 2016).

46 Although the effects of anesthesia on evoked potentials have been studied (Bithal 2014), the

47 presence of anesthetics might be a possible confounding factor and can alter the evoked

48 potentials. It is essential not to alter the pharmacological state of patients to avoid any changes in

49 the recording of evoked responses. It is also important to recognize the responses of evoked

50 potentials under anesthesia during clinical and experimental conditions. However, data

51 describing the effects of anesthesia on VEPs in avian animals under laboratory settings are still

52 insufficient.

53 Despite anesthetic agents affecting the amplitude and latency of evoked potentials through

54 direct inhibition of synaptic pathways or by indirectly altering the balance between inhibitory 
55 and excitatory influences (Bithal 2014; Sloan \& Jäntti 2008), evoked potentials obtained from

56 anesthetized monkeys (Nauhaus et al. 2009) were still shown to be valuable data compared to

57 those gathered from awake monkeys (Ray \& Maunsell 2011). Working under anesthetic

58 conditions is sometimes inevitable, especially when working with animals that cannot be

59 physically restrained. For example, chemical restraint is one of the options to make monkeys

60 watch a monitor for minutes without motion artifacts when studying their visual systems

61 (Nauhaus et al. 2009). If the monkeys are not chemically restrained, the monkeys need to be

62 trained over several months in order to record their brain activities of visual systems (Ray \&

63 Maunsell 2011). In addition, chemical restraint is helpful in avoiding interfering

64 electromyographic signals (Brauer et al. 2011; Itamoto et al. 2001) which may generate from eye

65 muscles or movements such as rotation of the eyeballs, blinking, and head turning (Brauer et al.

66 2011). Finally, it can minimize the stress caused by experimental procedures and provide

67 analgesia and autonomic reflex stability (Dondi et al. 2016; Pypendop et al. 1999).

68 Avian animals are visually inclined animals with well-developed color vision that have

69 attracted more attention in avian behavior, ecology, and neuroscience studies (Martin \& Osorio

70 2008). Among all of the avian animals, zebra finch (Taeniopygia guttata) is one of avian species 
71 that is commonly used as animal models as regard of neuroscience experiments especially in

72 studying the auditory system and language learning (Brainard \& Doupe 2002). In regard to

73 visual relative studies, researchers also reported that zebra finches are less interested in potential

74 mates with black beaks (Burley \& Coopersmith 1987; Collins et al. 1994). At least two visual

75 pathways, the tectofugal and thalamofugal pathway, are identified in avian animals (Zeigler et al.

76 1993). It will be a very intriguing question about how zebra finches use these visual pathways to

77 process color information in their brains (Bennett et al. 1996; Hunt et al. 1997). Analyzing VEPs

78 from the brain areas of these visual pathways may shed some lights on these questions. In an

79 ideal scenario, performing a surgery that implants electrodes in multiple brain areas and

80 conducting VEP experiments after the animal is fully recovered from anesthesia could explain

81 the questions best. However, technically, in order to record multiple brain regions of a fully

82 recovered zebra finch, carrying heavy implantation materials including electrodes and connectors

83 is mandatory and may raise ethical concern. To overcome above mentioned limitations, we

84 simplified the procedure by recording VEPs in unanesthetized birds' visual nucleus, rotundus

85 (ROT), which can process color information in tectofugal pathway of birds (Hodos 1969), and

86 compared the VEPs to anesthetized birds that were recorded immediately after implantation. We 
87 propose an anesthesia protocol that have acceptable effects on VEPs and can be applied on

88 multiple brain areas recording in zebra finches.

89 However, using anesthesia in birds is challenging. A study aiming at estimating the risks of

90 anesthetic and sedation-related mortality in companion animals found that the

91 anesthetic/sedation-related mortality in birds ranging from $1.76 \%$ to $16.33 \%$ depends on

92 different species which was significantly higher than those in dogs $(0.17 \%)$ and cats $(0.24 \%)$

93 (Brodbelt et al. 2008). It has been hypothesized that the birds' small size contributes to their

94 higher mortality risk due to higher surface area to volume ratios, high metabolic rates, and

95 trachea opening; the facts described above predispose them to complications like hypothermia,

96 hypoglycemia, and difficulties in maintaining a patent airway. Additionally, the lack of

97 experience of veterinary surgeons with exotic avian species was likely to have contributed to the

98 high perioperative mortality risks (Brodbelt et al. 2008). To reduce the mortality rate, balanced

99 anesthetic protocols (Ilkiw 1999), which refers to the use of a mixture of drugs, such that the

100 advantages of small amounts of drugs are used without having to contend with the disadvantages

101 of large doses of any one drug (Ilkiw 1999), are widely used in avian practice to increase the

102 safety of anesthetic procedures (Kubiak et al. 2016; Paula et al. 2013). Butorphanol, an opioid 
103 which is a mixed agonist/antagonist that has low activity at $\mu$-receptors and strong agonist

104 activity at $\kappa$ - receptors, makes it appropriate for avian species. The analgesic effect has been

105 validated in pigeons and psittacines. Midazolam has been used in avian species for sedation. It is

106 administered by intramuscular injection and has been demonstrated to have no significant

107 changes in cardiopulmonary function in Canadian geese (Branta canadensis), guinea fowl

108 (Numida meleagris), pigeons (Columba livia), or quail (Coturnix japonica) (Kubiak et al. 2016).

109 Opioids (Butorphanol) combined with benzodiazepines (Midazolam) are commonly used as

110 premedicants in birds before an inhalational agent such as isoflurane.

111 The objective of this study was to determine the effects of general anesthesia induced by

112 butorphanol-midazolam-isoflurane on intracranial evoked potentials in zebra finches by

113 comparing it to physically restrained zebra finches. The goal is to highlight the safety and

114 feasibility of this protocol for a relatively small animal under difficult anesthesia conditions, and

115 ultimately to provide a balanced anesthetic option in small experimental targets undergoing VEP

116 recording projects, and to test whether the protocol interferes VEP signals. The current study

117 compares VEPs from conscious and anesthetized states in zebra finches. 


\section{Materials \& Methods}

120 Animals

121 Eight male zebra finches (18-21 g) from a commercial breeder (San-Xing Bird Store,

122 Taipei, Taiwan) were used in these experiments. The animals were individually housed in home

123 cages with temperature controlled at $23 \pm 1^{\circ} \mathrm{C}$, and the light-dark cycle (AM 7:00 on, PM 7:00

124 off) was maintained under a natural photoperiod. Food and water were available ad libitum.

125 Commercial bird feed which contains millet kernel, canary millet, vitamins,

126 minerals (Ho Mei Chien, Taichung, Taiwan) was provided. All procedures performed in this

127 study were approved by the National Taiwan University Animal Care and Use Committee

128 numbered NTU106-EL-00026. Six birds were randomly assigned to one of two groups in which

129 flash-evoked potentials were monitored under physical (Bird 1, 5, 6) or chemical restraint by the

130 midazolam-butorphanol-isoflurane anesthesia (Bird 2, 3, 4). Additional control experiments were

131 performed on 2 of the 8 animals. Bird 7 was sedated by diazepam. Bird 8 was physically

132 restrained but its visual stimuli were blocked by a black ethylene-vinyl acetate (EVA) foam (30

$133 \mathrm{~cm} \times 25 \mathrm{~cm} \times 0.5 \mathrm{~cm} ;$ Yu Yuan Plastic, Taipei, Taiwan) 
135

136

137

138

139

140

141 Biotech Inc., Taoyuan, Taiwan) combined with 100\% oxygen that was provided by use of a

142 veterinary anesthesia delivery system (A.D.S 2000; ENGLER, Hialeah, Fla). The same

Surgery and Anesthesia

medication with intramuscular injection of $1 \mathrm{mg} / \mathrm{kg}$ midazolam (Dormicum, Roche Ltd,

Fontenay-sous-Bois, France) and $1 \mathrm{mg} / \mathrm{kg}$ butorphanol (Ilium Butorgesic injection, Troy

Laboratories Pty. Ltd, Australia) (Hawkins et al. 2018) into the pectoral muscle. Ten minutes

after injection, anesthesia was induced with 3\% isoflurane (Attane, Isoflurane, Panion \& BF

143

anesthetic delivery system was used to maintain anesthesia in a handmade face mask to maintain

144 a tight seal without blocking both eyes with 1.5-3\% isoflurane adjusted for the anesthetic depth

145 of the bird. The depth of anesthesia was determined through monitoring respiratory rate, corneal

146 reflex, palpebral reflex and wing withdrawal reflex (Heard 2015).

147

The skull of the bird was fixed on a stereotaxic frame (Stoelting, Wood Dale, IL) by gently

148

inserting earbars in its external acoustic canals. The coordinates of the target region, the nucleus

149 rotundus (ROT), were selected based on a stereotaxic atlas of the brain of the zebra finch

150 (Nixdorf-Bergweiler \& Bischof 2007). (AP, $2.6 \mathrm{~mm}$; ML, -2.0 mm; DV, $5.0 \mathrm{~mm}$ relative to y 
151 point). The tissue over the surface of the skull was removed and a hole was drilled for electrode

152 implantation. Before inserting the electrode, the dura was removed with a $27 \mathrm{G}$ needle. A tetrode

153 consisting of four twisted $0.05 \mathrm{~mm}$ stainless steel wires (California Fine Wire, Grover Beach,

154 CA) was slowly implanted into the nucleus rotundus (ROT), which is a visual nucleus that

155 processes color information in birds (Hodos 1969). Two stainless steel ground screws (M1.0*3

$156 \mathrm{~mm}$ ) were turned into the right frontal bone. The LFP from the ROT and the potential from right

157 hemisphere were connect to an interface board. For the birds (Birds 2 to 4 ) that were recorded

158 under anesthesia, the interface board was linked to a 20X-gain head stage and tethered to the

159 preamplifier of an OmniPlex A system (Plexon, Dallas, TX, USA). Otherwise, the interface

160 board was cemented to the skull with dental acrylic (Tempron, GC Co., Tokyo, Japan), and the

161 subject had recovered for 7-10 days before LFP and VEP recording. Postoperatively, birds in the

162 experimental group were euthanized after completed experimental procedures described in the

163 following section. For the control group, $5 \mathrm{mg} / \mathrm{kg}$ carprofen (Pfizer, New York, USA) was

164 provided by intramuscular injection for 3 days for postoperative analgesia.

165 
168 physically restrain five of the subjects (Bird 1, 5, 6, 7, 8) and then applied a small amount of

169 Cyanoacrylate (Loctite 415, Henkel, Germany) on the right eyelid, and the subject was placed in

170 front of a laptop (model: SVP132A1CP, SONY, Japan) with the right eye aimed at the screen

171 (Fig. 1, illustrations). The color stimulation slides were made by Microsoft PowerPoint (2016;

172 Microsoft, Washington, USA). In order to further confirm the VEPs were not from non-specific

173 noises such as the static when changing slides, a black high density EVA foam which reflect

174 very little light was used to block the color stimuli from the screen (Bird 8). The foam was

175 lowered and elevated by hands (Fig. S1). For chemically restrained birds (Bird 2 to 4), after the

176 surgical procedure described above, \#6-0 surgical sutures were used to open and fix the right

177 eyelids. During the recording, the concentration of isoflurane was turned to $1 \%$. The grounding

178 screw was used as a reference, and the LFP signals were amplified (gain 2,500×), digitized,

179 bandpass-filtered (between 0 and $500 \mathrm{~Hz}$ ), and recorded (at $2000 \mathrm{~Hz}$ sampling rate) by a 16-

180 channel OmniPlex A system (Plexon, Dallas, TX, USA). All recording was performed in a

181 Faraday cage that was grounded with the amplifier. The laptop screen was approximately $30 \mathrm{~cm}$

182 away from the subject and placed outside the Faraday cage without plugging in the charger. The 
183 retinas of avian animals contain four kinds of cone cells and result in their tetrachromatic color

184 vision (Osorio et al. 1999; Viets et al. 2016). The cone cells are sensitive to the wavelengths

185 which are believed to be red, green, blue, and violet to ultraviolet. As a pilot study, we first

186 flashed 20 to 21 trials of 2 seconds of light green (RGB color code: [ $0.25,1,0.75$ ], 1x: 79E)

187 between 2 seconds of black (RGB color code: [ 0, 0, 0 ], lx: 2.4E) for Bird 1. The same

188 procedures were also done by flashing light red (RGB color code: [ 1, 0.25, 0 ], lx: 23E), and

189 light blue (RGB color code: [ 0, 0.75, 1 ], 1x: 45E) for Bird 1. We selected green as the color

190 stimuli for further experiments since it generated the largest VEPs (see Results section). The time

191 points switching between displaying color or black were saved in the same LFP acquisition file.

192 We also tested the VEPs under the effects of classic sedative agent which commonly used in

193 zebra finch: diazepam (Wolf et al. 2017). Bird 7 was used to test the effects diazepam. $4 \mathrm{mg} / \mathrm{kg}$

194 diazepam (Astar, Hsinchu, Taiwan) was administrated intramuscularly 10 minutes before

195 recording. A towel was rolled around its body for restraining (Fig. S2).

196

197 LFP Analysis 
202 site for each bird is difficult to control. Thus, the amplitudes of each \pm 1 -second LFP were Z-

203 scored across time for better comparison between subjects (Fig. 2A to 2D). The spectra of raw

204 data (spectra of Figs. 3, 4) were calculated by multitaper spectral analysis, and the error bars

205 were analyzed by the Jackknife method, using the mtspectrumc.m function in Chronux. We set

206 the time-bandwidth product and the number of leading tapers to use to 3 and 5, respectively. The

207 spectrograms (Fig. 4A to 4D) were similar to the spectrum but were further investigated in the

208 time domain. We used 0.5-sec windows with 0.05-sec overlapping steps, and the time-bandwidth

209 product of the number of tapers are the same as the spectra. The power of the LFP spectrum is

210 correlated with 1 /frequency (Buzsaki 2006), which results in low powers of high frequencies. To

211 compensate for this noise, we Z-scored every frequency along the time domain and then

212 Gaussian-smoothed the figure using the boxcar method.

\section{Exclusion criteria and data preprocessing}


214 Because the response time of VEPs may be interfered by the drugs, using computer programs to

215 exclude data with defined onset period may be not practical. To solved this, we defined the

216 exclusion criteria by both inspection of the onset of VEPs visually and set an amplitude threshold

217 for including the birds with VEPs. The procedures were done as follows: Each \pm 1 -second raw

218 LFP trace was stacked and plotted (Fig. 1, Figs. S1 to S4) for observing the VEPs. The birds with

219 repeatedly showing shooting LFPs after color stimuli were included in the study. To be specific,

220 the delta amplitudes of shooting LFPs were greater than $0.15 \mathrm{mv}$ when compared to the

221 amplitudes at 0 second. The LFPs of excluded birds were further pre-processed with independent

222 component analysis (ICA) for extract the potential data that masked by noises (Figs. S3 to S5).

223 An open source tool box, FastICA (Gävert et al. 2005) (Available at

224 https://research.ics.aalto.fi/ica/fastica/code/dlcode.shtml ), was used for the ICA. Two control

225 birds (Bird 5 and 6) were pre-processed and data were shown in Figs. S3 to S5.

\section{Statistics}

227 Recorded data were evaluated for the quality of the waveforms according to the raw LFP

228 traces. The bird with LFPs that showed enhanced amplitudes to light stimuli were included in the

229 statistics analysis. All results in the figures are depicted as the means \pm SEM. The statistical 
230 analyses were conducted with SPSS (Version: 10.0.7, IBM, Armonk, New York). For the

231 significant differences of spectra for each frequency (Figs 3 and 4E), the confidence bands at $p=$

2320.05 were calculated by the Jackknife method, using the mtspectrumc.m function in Chronux.

233 This gave the 95\% confidence intervals of the spectra (Bokil et al. 2010). Detailed methods are

234 described in the Results section. We used one-way ANOVA to analyze significant differences for

235 the latencies and peak amplitudes. A level of $p<0.05$ was taken as indicating a statistically

236 significant difference. 


\section{Results}

238 The drugs prolong the latency of VEP

239 Electrodes were implanted into the ROT in 8 zebra finches. In the control group, the

240 electrodes were glued to the birds' skulls, and the LFPs were recorded after 7-10 days of

241 convalescence. However, only 1 bird (Bird 1) showed waveforms that met the preset criteria

242 (Fig. 1, Figs. S3 to S4) for further analysis. In the experimental group, VEPs were recorded

243 during the implantation surgery under anesthesia. The raw LFP traces of anesthetized birds (Fig.

244 1A and 1C) showed a predominance of low frequencies, which represented smoother LFPs (Fig.

$2451 \mathrm{~A}$ vs $1 \mathrm{~B}$, before the light stimulation). Then, the visual pathway was further stimulated with

246 light green (color bar at the bottom of Fig. 1B) because we found that zebra finches are sensitive

247 to this color (z-scored VEP peak amplitudes for green: $2.8 \pm 0.2$, blue: $2.2 \pm 0.2$, red: $0.8 \pm 0.2$ in

248 Bird 1). The examples revealed that the latency of VEPs was longer in the experimental group

249 than in towel-restrained birds (Arrows, Fig. 1A vs Fig. 1B).

250 We further analyzed the LFPs of every trial. The impedances for each bird were not the

251 same, and they consequently affected the amplitude of the LFP. Therefore, we z-scored the LFPs

252 and averaged across every stimulus (Fig. 2A to 2D). The evoked potentials spiked at $415.9 \pm$ 
$25320.8 \mathrm{~ms}$, which was significantly longer compared to control birds (Fig. 2E, $197.9 \pm 7.3 \mathrm{ms,}$

$\left.254 \mathrm{~F}_{(1,80)}=40.8, p<0.01\right)$. In contrast, the amplitudes of evoked potentials were similar between the

255 chemically $(3.7 \pm 0.2)$ and physically $(3.3 \pm 0.2)$ restrained birds (Fig. $\left.2 \mathrm{~F}, \mathrm{~F}_{(1,80)}=1.7, p=0.19\right)$.

256 This finding suggested that acute recording (recording right after implantation) was not different

257 from delayed recording in terms of the amplitudes of VEPs. Similar to Fig. 1, the duration of

258 VEPs was also longer in chemically restrained birds than in physically restrained birds (Fig. 2A

259 vs $2 \mathrm{~B}$ to $2 \mathrm{D})$. We further salvaged the control data which have weak VEPs when inspected by

260 raw LFP traces (B5; Fig. S3 blue traces, B6; Fig. S4 blue traces). An ICA pre-processing was

261 applied on the raw LFPs of Bird 5 and Bird 6 (B5; Fig. S3 red traces, B6; Fig. S4 red traces).

262 Although the VEPs were not obvious, their z-scored traces, spectrograms, and ICA processed

263 spectrograms still represent VEP signals (Fig. S5). The z-scored amplitude were $1.3 \pm 0.3$ at 190

$264 \mathrm{~ms}$ in Bird 5 and $1.7 \pm 0.2$ at $216 \mathrm{~ms}$ in Bird 6 (Fig. S5A and D).

265 We were also curious about whether the VEPs were from some nonspecific artifacts which

266 generated by switching between the color and black slides in the screen. Thus, we used a black

267 high density EVA foam to physically block the green light from the screen and conduct a towel

268 restrained experiment again (Bird 8; Fig. S1). The VEPs were still generated (Fig. S1A and B) 
269 although the waveforms are wider than Bird 1 (Fig. 2A). This phenomenon can be explained by

270 the speed limitation for lowing and elevating the foam by hands when compared with the snap

271 changes of slides by PowerPoint.

272

273 The drugs decreased overall power but not VEPS

$274 \quad$ For further inspecting which bands were mainly affected, we cut the raw LFP traces into \pm

275 1-second segments starting from the flash time points, which represent zero (see Materials \&

276 Methods: LFP Analysis). Then, the LFPs were transformed into pre- and poststimulus spectra

277 (prestimulus: Fig. 3A to 3C; poststimulus: Fig. 3D to 3E). The 95\% confidence intervals of the

278 spectra were showed by the shadows and bands. Figure 3 displays that the frequencies $>5 \mathrm{~Hz}$

279 were diminished by the drugs, but frequencies $<5 \mathrm{~Hz}$ were unaffected, which is consistent with

280 the results observed from LFP traces (Fig. 1 and Fig. 2A to 2D). These spectra suggested that the

281 local computations of neurons that oscillate at high frequency were abolished by the drugs

282 (Buzsaki \& Schomburg 2015; Zheng et al. 2016), but the ROT still generated the brain waves

283 that synchronized with other brain regions. When inspecting the 1-second spectra after stimuli

284 (Fig. 3D to 3F), we noticed that changes ( $\Delta$ power) between the physically and chemically 
285 restrained birds were decreased. Thus, we analyzed the time domain of the spectrum to illustrate

286 the spectrograms (Fig. 4A to 4D). The dynamics of the spectra echoed the results in Fig. 3A to

$2873 \mathrm{C}$, which showed relatively weak power before light stimulation compared to the physically

288 restrained bird. However, the power of VEPs were not diminished by drugs (Fig. 4C to 4D

289 between dashed lines). Therefore, we cut the LFP traces between the VEPs (Fig. 4A to 4D

290 between dashed lines) and calculated their spectra (Fig. 4E). Interestingly, by comparing the

291 confidence interval of the spectra, the frequencies below $30.8 \mathrm{~Hz}$ (Fig. 4E between solid lines)

292 were significantly stronger in chemically restrained finches. The data implied that although

293 midazolam-butorphanol-isoflurane generally suppressed the functions of local networks (Buzsaki

294 \& Schomburg 2015; Zheng et al. 2016), light cues still triggered strong evoked potentials (Fig.

295 4E), suggesting that the anesthesia protocol is feasible for recording evoked potentials.

296 In addition to testing this three-agent anesthesia protocol for further multi-regional intracranial

297 recording, we were also interested in comparing the influences of the traditional sedative,

298 diazepam. Diazepam increases the effects of the inhibitory neurotransmitter gamma-

299 aminobutyric acid (GABA) (Riss et al. 2008) and can be used as a sedative for zebra finches in

300 order to record activities of auditory systems (Wolf et al. 2017). Diazepam (4 mg/kg) provides 
301 only a sedative effect instead of an anesthetic effect (Heard 2015) so it can not be used alone

302 during an acute implantation recording. For this reason, we test the effects of diazepam in a

303 towel restrained zebra finch. Fig. S2 shows that $4 \mathrm{mg} / \mathrm{kg}$ diazepam did not suppress the

304 amplitudes of LFPs when compared to general anesthesia (Figs. 1, 2, 4; Bird 2-4). Moreover, the

305 latency of VEP was not interfered by $4 \mathrm{mg} / \mathrm{kg}$ diazepam (peaked at $174.5 \mathrm{~ms} ;$ Fig. S2A).

306 


\section{Discussion}

308

Designation of the present anesthetic protocol

309

The clinical usefulness of flash VEPs is well known in human medicine because it allows

310

objective assessment of the functional integrity of the visual pathways from the retina to the

311 visual cortex, even during general anesthesia or coma conditions (Dondi et al. 2016). However, a

312 few decades ago, VEPs could not be reliably interpreted intraoperatively, and lack of consistency

313 made VEPs a less effective monitoring tool than other modalities during anesthesia, which

314 suggested variable influence on the potential by anesthetic agents (Cedzich \& Schramm 1990).

315 Therefore, successful evoked potential monitoring requires an adequate understanding of how

316 anesthetic drugs and physiological variations affect signals and how to improve the sensitivity of

317 neuromonitoring through appropriate drug selection and administration (Soghomonyan et al.

318 2014). Evoked potentials are highly sensitive to fluctuations in physiological parameters, such as

319 peripheral and core body temperature, arterial blood pressure, hematocrit, and others

320 (Soghomonyan et al. 2014). VEPs are the most sensitive sensory evoked potential to anesthetics

321 in general because the evoked responses travel via polysynaptic pathways (Kumar et al. 2000;

322 Soghomonyan et al. 2014). In general, inhalational anesthetics are more potent suppressors of

Peer] reviewing PDF | (2019:04:36984:2:0:NEW 11 Sep 2019) 
323 evoked potentials than intravenous agents. Therefore, balanced general anesthesia with low

324 doses of inhalational agents combined with injectable agents may be recommended

325 (Soghomonyan et al. 2014).

326 To reduce the dose of inhalational anesthetics and therefore avoid its' suppression effects on

327 evoked potentials, a balanced anesthetic protocol was selected in the current study to provide a

328 stable physiological response in anesthetized avian patients and create a less sensitive anesthetic

329 and proper analgesic combination for VEP monitoring. Opioids are known to alter sensitive

330 evoked potentials minimally compared to inhalation agents (Soghomonyan et al. 2014).

331 Schwender et al. demonstrated that opioids produce powerful analgesia and have little or no

332 effect on middle latency auditory-evoked potentials (MLAEP) (Schwender et al. 1993). In

333 humans, midazolam at induction doses $(0.2 \mathrm{mg} / \mathrm{kg})$ in the absence of any other agent results in

334 slight depression of cortical somatosensory evoked potential amplitude and has minimal effect

335 on subcortical and peripheral components. However, adding an opioid to midazolam preserves

336 cortical somatosensory evoked potentials better compared with adding opioids to thiopentone or

337 etomidate (Bithal 2014). Combining isoflurane increases flexibility and safety in the protocol as

338 anesthetic depth can be adjusted instantaneously according to the individual's physiologic 
339 response during the procedure (Heard 2015). In prior research, the midazolam-butorphanol

340 combination demonstrated an isoflurane-sparing effect in birds, which minimizes side effects

341 caused by isoflurane during anesthesia and during VEP monitoring (Curro 1994; Curro et al.

342 1994). Therefore, a balanced anesthetic protocol was finally designed considering both the safety

343 of the avian animals and the representativeness of the evoked potentials.

345 Effects of anesthetic combination

346 Few studies have focused on the effect of anesthetics on flash VEPs in humans (Kumar et

347 al. 2000) or animals. A pilot study conducted in birds of prey showed that both the peak latency

348 and wave morphology from normal animals obtained solely under isoflurane anesthesia were

349 similar to those obtained previously in other animal species (Dondi et al. 2016). This test can be

350 easily and safely performed in a clinical setting in birds of prey and could be useful for an

351 objective assessment of visual function (Dondi et al. 2016). However, there was no control group

352 to describe the effects of isoflurane on the VEPs of birds of prey.

353 The increased latency in our current study is comparable to the finding that administration

354 of midazolam-butorphanol tended to increase the latency of middle latency auditory-evoked 
355 potentials due to its' induction of profound neuroleptanalgesia (Pypendop et al. 1999).

356 Furthermore, isoflurane tends to increase the latency and decrease the amplitudes of VEPs

357 (Kumar et al. 2000). We speculated that the threshold of action potentials may be increased by

358 anesthetics (Ries \& Puil 1999), resulting in difficulty with fast responses (Brown et al. 2010),

359 and finally delay the VEPs. However, the power of the VEPs below $30.8 \mathrm{~Hz}$ were not diminished

360 but were enhanced. If we observe the LFP without the VEPs, suppression of high frequency field

361 potentials (Fig. 2A to 2D, Fig. 4A to 4D) is common in anesthetized subjects (Hagihira et al.

362 2002; Purdon et al. 2013) or animals in deep sleep stages (Murphy et al. 2011). Reports shows

363 that frequencies above $10 \mathrm{~Hz}$ are suppressed by anesthetics (Hagihira et al. 2002; Purdon et al.

364 2013). We demonstrated that the VEPs with frequencies below $30.8 \mathrm{~Hz}$ were more prominent

365 after chemical restraint (Fig. 4E). We think it is because the sedatives and anesthetics improve

366 the signal-to-noise ratio (Banoub et al. 2003; Brauer et al. 2011; Sloan 1994) and the VEPs from

367 subcortical recording method is less sensitive to anesthetics (Banoub et al. 2003). Firstly, the

368 sedatives and anesthetics reduced movement artifacts or other electromyography artifacts (they

369 usually introduce noise at higher frequencies) that normally mask the evoked potentials of

370 physical restrained animals (Banoub et al. 2003; Brauer et al. 2011; Sloan 1994). Secondly, 
371 subcortical activities are less sensitive to anesthetics when compared to cortical activities

372 because the cortical activities are usually involved in polysynaptic pathways (Banoub et al.

373 2003), and the anesthetics predominantly act on synaptic transmission (Banoub et al. 2003;

374 Richards 1983). Combining the two advantages made VEPs more prominent and easier to

375 observe.

376

377 Alternative anesthetic options

378 Recent studies have employed total intravenous anesthesia during VEP monitoring. The

379 apparent improvement in recording VEPs intraoperatively when switching from inhalational

380 anesthesia to total intravenous anesthesia (TIVA) led investigators to examine the role of other

381 anesthetic agents (Sharika et al. 2016). Among these, dexmedetomidine, an $\alpha-2$ agonist, which

382 differs from other anesthetics by its non- $\gamma$ - aminobutyric acid (GABA) mechanisms of sedation

383 and anxiolysis, was studied as an adjunct to TIVA. If baseline VEPs can be obtained in a patient,

384 then dexmedetomidine does not interfere with the acquisition of intraoperative VEPs (Rozet et

385 al. 2015). Although TIVA improved the chances of recording intraoperative VEPs reliably, it is

386 impossible to administer TIVA in zebra finches due to their small size. An intraosseous route 
387 with a very delicately calibrated syringe pump might be an anesthetic option in the future

388 (Briscoe \& Syring 2004). An unignorable fact is the profound cardiovascular side effects

389 (Hornak et al. 2015). The safety of using dexmedetomidine in the already challenging avian

390 anesthesia should be considered, especially when the dose range of dexmedetomidine for finches

391 is not known currently (Hawkins et al. 2018).

392

393

Comparison of waveform performance

394

In a previous study in bird of prey (Dondi et al. 2016), the waveforms of VEPs were

395

complex since they used electroencephalography by applying electrodes under the skin of the

396 skull, which obtain the signals from relatively large areas. In contrast, we recorded LFPs from

397 the ROT, which receives signals from the optic nerve and is the first relay area of the visual

398 pathway. By analyzing VEPs in this area, the data should be more precise to reflect the effect of

399 drugs when compared with physically restrained subjects, for which it is difficult to eliminate

400 artifacts from muscles when using skull electroencephalography. Despite this issue, a nonsedated

401 brain may inhibit the ROT and give weaker VEPs (Fig. 4E). 
403 Limitations and future work

404 The limitations of the current study included that an ophthalmic examination and

405 neurologic exam was not conducted prior to the experiment due to the small size of the finch.

406 However, there was no obvious ophthalmic defect or visual impairment observed before starting

407 the experiment. There has been little discussion in the literature of anesthetic effects on purely

408 visual evoked potentials in birds. Therefore, our anesthetic protocol was created based on reports

409 of either sensory evoked potentials or MLAEP. A similar situation was found again when

410 discussing anesthetic effects in the current study. The proposed effects of anesthetics including

411 increased peak latencies, suppression of high frequency waves were supported mainly based on

412 those concluded in existing reports of mammals (Kumar et al. 2000; Nauhaus et al. 2009). It

413 should be further verified in avian animals and with a well-controlled study in the future.

\section{Conclusions}

415 In the current study, zebra finches were given midazolam-butorphanol-isoflurane and had

416 VEPs recorded to determine a balanced anesthetic protocol. Our results demonstrate that flash

417 VEP could be recorded under the protocol because VEP power below $30.8 \mathrm{~Hz}$ did not weaken 
418 with chemical restraint (Fig. 5). On the other hand, our results showed that physical restraint in

419 small animals was not ideal due to the data from physically restrained birds being difficult to

420 acquire as a result of motion artifacts and the attention of the bird. We repeated the procedure in

421 three birds, but only one bird showed VEPs. Finally, the lower power of VEPs (below $30.8 \mathrm{~Hz}$ )

422 of the physically restrained bird (Fig. 4E) may be caused by the summation of voltage

423 fluctuations of several active brain areas which result in a less synchronized status (Ray \&

424 Maunsell 2011). As mentioned previously, LFP data contains more information when recorded

425 in several brain regions simultaneously but it is not as easy to accomplish in zebra finches owing

426 to their high mortality rate during surgery. Although there was minor interference of the received

427 signal (delayed evoked potentials) with the current anesthetic protocol, the benefits (such as

428 application to multi-regional recording in avian animals) were enough to compensate for those

429 drawbacks. Therefore, it is recommended that VEPs be recorded in small animals under

430 anesthesia, and the anesthetic protocol and basic VEP waveform of the zebra finch under the

431 midazolam-butorphanol-isoflurane anesthesia presented in the current study can be applied for

432 similar electrophysiological studies and clinical applications in other species. 


\section{Acknowledgements}

435 The authors thank National Taiwan University Veterinary Hospital for technically supporting the

436 study. This study was supported by grants from Ministry of Science and Technology

437 (MOST107-2311-B-002-005 and MOST105-2911-I-002-520). 
438

439 References

440 Banoub M, Tetzlaff JE, and Schubert A. 2003. Pharmacologic and physiologic influences

441 affecting sensory evoked potentials: implications for perioperative monitoring.

442

Anesthesiology 99:716-737. 10.1097/00000542-200309000-00029

443 Bennett ATD, Cuthill IC, Partridge JC, and Maier EJ. 1996. Ultraviolet vision and mate choice

in zebra finches. Nature 380:433. 10.1038/380433a0

445 Bithal P. 2014. Anaesthetic considerations for evoked potentials monitoring. Journal of

Bokil H, Andrews P, Kulkarni JE, Mehta S, and Mitra PP. 2010. Chronux: a platform for analyzing neural signals. J Neurosci Methods 192:146-151. 358. $10.1038 / 417351 \mathrm{a}$ recordings in dogs: Prevention of muscle artifacts and evaluation of two activation techniques in healthy individuals. Res Vet Sci 90:306-311. 10.1016/j.rvsc.2010.06.004 
455 Briscoe JA, and Syring R. 2004. Techniques for emergency airway and vascular access in special

456

457

458

459

460

461

462

463

464

465

466

467

468

469

470 species. Seminars in Avian and Exotic Pet Medicine 13:118-131. 10.1053/j.saep.2004.03.007

Brodbelt DC, Blissitt KJ, Hammond RA, Neath PJ, Young LE, Pfeiffer DU, and Wood JL. 2008. The risk of death: the confidential enquiry into perioperative small animal fatalities. Vet Anaesth Analg 35:365-373. 10.1111/j.1467-2995.2008.00397.x

Brown EN, Lydic R, and Schiff ND. 2010. General anesthesia, sleep, and coma. N Engl J Med 363:2638-2650. 10.1056/NEJMra0808281

Burley N, and Coopersmith CB. 1987. Bill Color Preferences of Zebra Finches. Ethology 76:133-151. 10.1111/j.1439-0310.1987.tb00679.x

Buzsaki G. 2006. Rhythms of the Brain: Oxford University Press.

Buzsaki G, and Schomburg EW. 2015. What does gamma coherence tell us about inter-regional neural communication? Nature Neuroscience 18:484. 10.1038/nn.3952

Cecchetto C, Mahmud M, and Vassanelli S. 2015. Anesthesia effect on single local field potentials variability in rat barrel cortex: Preliminary results. Conf Proc IEEE Eng Med Biol Soc 2015:4721-4724. 10.1109/EMBC.2015.7319448 
471 Cedzich C, and Schramm J. 1990. Monitoring of flash visual evoked potentials during

472 neurosurgical operations. Int Anesthesiol Clin 28:165-169.

473 Collins SA, Hubbard C, and Houtman AM. 1994. Female mate choice in the zebra finch — the

474 effect of male beak colour and male song. Behavioral Ecology and Sociobiology 35:21-

$475 \quad 25.10 .1007 / \mathrm{bf00167055}$

476 Curro T. 1994. Evaluation of the isoflurane-sparing effects of butorphanol and flunixin in

477 psittaciformes. Proc Annu Conf Assoc Avian Vet.

478 Curro TG, Brunson DB, and Paul-Murphy J. 1994. Determination of the ED50 of isoflurane and

479 evaluation of the isoflurane-sparing effect of butorphanol in cockatoos (Cacatua spp.).

$480 \quad$ Vet Surg 23:429-433.

481 Dondi M, Biaggi F, Di Ianni F, Dodi PL, and Quintavalla F. 2016. Flash visual evoked potentials

482 in diurnal birds of prey. PeerJ 4:e2217. 10.7717/peerj.2217

483 Gävert H, Hurri J, Särelä J, and Hyvärinen A. 2005. FastICA 2.5. Available at https://researchicsaaltofi/ica/fastica/code/dlcodeshtml.

485 Hagihira S, Takashina M, Mori T, Mashimo T, and Yoshiya I. 2002. Changes of electroencephalographic bicoherence during isoflurane anesthesia combined with 
epidural anesthesia. Anesthesiology 97:1409-1415.

488 Hawkins MG, Guzman DSM, Beaufrere H, Lennox AM, and Carpenter JW. 2018. Birds. In: Carpenter JW, ed. Exotic Animal Formulary. St. Louis, Missouri: Elsevier, 167-375.

490

491

492

493

494

495

496

497

498

499

500

501

Heard D. 2015. Anesthesia. In: Speer B, ed. Current Therapy in Avian Medicine and Surgery Elsevier Health Sciences, 601-615.

Hodos W. 1969. Color Discrimination Deficits After Lesions of the Nucleus Rotundus in Pigeons. Brain, Behavior and Evolution 2:185-200. 10.1159/000125822

Hornak S, Liptak T, Ledecky V, Hromada R, Bilek J, Mazensky D, and Petrovic V. 2015. A preliminary trial of the sedation induced by intranasal administration of midazolam alone or in combination with dexmedetomidine and reversal by atipamezole for a short-term immobilization in pigeons. Vet Anaesth Analg 42:192-196. 10.1111/vaa.12187

Hunt S, Cuthill IC, Swaddle JP, and Bennett ATD. 1997. Ultraviolet vision and band-colour preferences in female zebra finches, Taeniopygia guttata. Anim Behav 54:1383-1392.

Ilkiw JE. 1999. Balanced anesthetic techniques in dogs and cats. Clin Tech Small Anim Pract $14: 27-37.10 .1016 / \mathrm{S} 1096-2867(99) 80024-3$ 
503

504

505

506

507

508

509

510

511

512

513

514

515

516

517

medetomidine on electroencephalography and use of a quantitative

electroencephalograph for evaluating sedation levels in dogs. J Vet Med A Physiol Pathol

Clin Med 48:525-535.

Kubiak M, Roach L, and Eatwell K. 2016. The Influence of a Combined Butorphanol and Midazolam Premedication on Anesthesia in Psittacid Species. J Avian Med Surg 30:317-

323. $10.1647 / 2013-072$

Kumar A, Bhattacharya A, and Makhija N. 2000. Evoked potential monitoring in anaesthesia and analgesia. Anaesthesia 55:225-241.

Martin GR, and Osorio D. 2008. The senses. In: Masland RH, and Albright TD, eds. Vision in birds. London: Academic Press, 25-52.

Murphy M, Bruno MA, Riedner BA, Boveroux P, Noirhomme Q, Landsness EC, Brichant JF, Phillips C, Massimini M, Laureys S, Tononi G, and Boly M. 2011. Propofol anesthesia and sleep: a high-density EEG study. Sleep 34:283-291A.

Nauhaus I, Busse L, Carandini M, and Ringach DL. 2009. Stimulus contrast modulates functional connectivity in visual cortex. Nat Neurosci 12:70-76. 10.1038/nn.2232

518 Nixdorf-Bergweiler BE, and Bischof H-J. 2007. A stereotaxic atlas of the brain of the zebra 
519

520

521

522

523 Paula VV, Otsuki DA, Auler Junior JO, Nunes TL, Ambrosio AM, and Fantoni DT. 2013. The

524

525

526

527

528

529

530

531

532

533

534

finch, Taeniopygia guttata, with special emphasis on telencephalic visual and song system nuclei in transverse and sagittal sections. Bethesda (MD).

Osorio D, Vorobyev M, and Jones CD. 1999. Colour vision of domestic chicks. J Exp Biol 202:2951-2959.

effect of premedication with ketamine, alone or with diazepam, on anaesthesia with sevoflurane in parrots (Amazona aestiva). BMC Vet Res 9:142. 10.1186/1746-6148-9-142

Picton TW, Bentin S, Berg P, Donchin E, Hillyard SA, Johnson R, Jr., Miller GA, Ritter W, Ruchkin DS, Rugg MD, and Taylor MJ. 2000. Guidelines for using human event-related potentials to study cognition: recording standards and publication criteria. Psychophysiology 37:127-152.

Purdon PL, Pierce ET, Mukamel EA, Prerau MJ, Walsh JL, Wong KF, Salazar-Gomez AF, Harrell PG, Sampson AL, Cimenser A, Ching S, Kopell NJ, Tavares-Stoeckel C, Habeeb K, Merhar R, and Brown EN. 2013. Electroencephalogram signatures of loss and recovery of consciousness from propofol. Proc Natl Acad Sci U S A 110:E1142-1151.

$10.1073 /$ pnas. 1221180110 
535 Pypendop B, Poncelet L, and Verstegen J. 1999. Use of midlatency auditory-evoked potentials as

536

537

538

539

540

541

542

543

544

545

546 Riss J, Cloyd J, Gates J, and Collins S. 2008. Benzodiazepines in epilepsy: pharmacology and

547

548

549

550

indicator of unconsciousness in the dog: characterisation of the effects of acepromazine-

thiopentone, medetomidine-thiopentone and medetomidine-butorphanol-midazolam

combinations. Res Vet Sci 67:35-39. 10.1053/rvsc.1998.0273

Ray S, and Maunsell JH. 2011. Network rhythms influence the relationship between spiketriggered local field potential and functional connectivity. J Neurosci 31:12674-12682.

10.1523/JNEUROSCI.1856-11.2011

Richards CD. 1983. Actions of general anaesthetics on synaptic transmission in the CNS. $B r J$

Anaesth 55:201-207. 10.1093/bja/55.3.201

Ries CR, and Puil E. 1999. Mechanism of anesthesia revealed by shunting actions of isoflurane on thalamocortical neurons. J Neurophysiol 81:1795-1801. 10.1152/jn.1999.81.4.1795

Rozet I, Metzner J, Brown M, Treggiari MM, Slimp JC, Kinney G, Sharma D, Lee LA, and pharmacokinetics. Acta Neurol Scand 118:69-86. 10.1111/j.1600-0404.2008.01004.x Vavilala MS. 2015. Dexmedetomidine Does Not Affect Evoked Potentials During Spine Surgery. Anesth Analg 121:492-501. 10.1213/ANE.0000000000000840 
551 Schwender D, Rimkus T, Haessler R, Klasing S, Poppel E, and Peter K. 1993. Effects of

552 increasing doses of alfentanil, fentanyl and morphine on mid-latency auditory evoked

$553 \quad$ potentials. Br J Anaesth 71:622-628.

554 Sharika R, Mirela V, and Dinesh G. 2016. Intraoperative Visual Evoked Potentials: There is

$555 \quad$ More to it than Meets the Eye. Journal of Neurology and Neuroscience 7:106-117.

556

$10.21767 / 2171-6625.1000106$

557 Sloan TB. 1994. Nondepolarizing neuromuscular blockade does not alter sensory evoked

558 potentials. J Clin Monit 10:4-10.

559 Sloan TB, and Jäntti V. 2008. Anesthetic effects on evoked potentials. Handbook of Clinical

$560 \quad$ Neurophysiology: Elsevier, 94-126.

561 Soghomonyan S, Moran KR, Sandhu GS, and Bergese SD. 2014. Anesthesia and evoked

562 responses in neurosurgery. Front Pharmacol 5:74. 10.3389/fphar.2014.00074

563 Viets K, Eldred K, and Johnston RJ, Jr. 2016. Mechanisms of Photoreceptor Patterning in

564 Vertebrates and Invertebrates. Trends Genet 32:638-659. 10.1016/j.tig.2016.07.004

565 Wolf SE, Swaddle JP, Cristol DA, and Buchser WJ. 2017. Methylmercury Exposure Reduces the Auditory Brainstem Response of Zebra Finches (Taeniopygia guttata ). J Assoc Res 
Otolaryngol 18:569-579. 10.1007/s10162-017-0619-7

568 Zeigler HP, Bischof HJ, and Technology MIo. 1993. Vision, Brain, and Behavior in Birds: MIT

$569 \quad$ Press.

570 Zheng C, Bieri KW, Hsiao YT, and Colgin LL. 2016. Spatial Sequence Coding Differs during

$571 \quad$ Slow and Fast Gamma Rhythms in the Hippocampus. Neuron 89:398-408.

$572 \quad$ 10.1016/j.neuron.2015.12.005

573

$574 \quad$ Figure 1. Examples of raw LFP traces after watching a light under chemical or physical

575 restraint. (A) and (B) are graphical abstracts showing the method for recording the evoked

576 potentials. The zebra finches were either restrained by intramuscular administration of

577 midazolam and butorphanol and maintained by inhalation of isoflurane or rolled with a

578 towel. The electrodes were inserted into the left ROT and recorded the LFP while the right

579 eye watched the screen. The screen displayed blacks and greens. (A) is an example of LFP

580 and the evoked potentials of a chemically restrained bird. (B) was obtained with towel

581 restraint. (C) and (D) are ten examples of LFP raw traces. The vertical lines and the arrow

582 heads at zeros are the time points when the black switched to green. 
583

584

585

586

587

588

589

590

591

592

593

594

595

596

597
Figure 2. (A) to (D) Averaged LFP traces for each subject. The illustration in each figure shows the restraint method. The LFPs were normalized by standard deviation and represented with Z-scores. (E) is averaged peak latencies. The Z-scores for peaks are averaged in $(\mathrm{F})$. The lines and shadows and bars and error bars are means $\pm \mathrm{SEM}$. Zero second means the time when the screen changed color from black to green. $*$ denotes the $p$ value $<0.01$

Figure 3. (A) to $(\mathrm{C})$ are spectra from one-second LFPs when watching blacks (e.g., -1 to 0 second). (D) to (F) are data from one-second LFP bins after the black changed to green (e.g., 0 to 1 second). The white, red, and blue bands were means $\pm 95 \%$ confidence intervals from Birds 2, 3, and 4 respectively, which were chemically restrained finches. The gray bands are from the physically restrained bird (Bird 1) and illustrate the $95 \%$ confidence intervals. For comparison, the spectra of the physically restrained bird were plotted with the chemically restrained birds. 
598

599

600

601

602

603

604

605

606

607

608

609

610

611

612

Figure 4. (A) to (D) Averaged spectrogram of each subject. The warmer color indicates

stronger Z-scored power at a specific frequency (y-axis) during a specific time (x-axis). The spectra between the dashed lines are displayed in (E) and are a comparison between bird 1 (shadow) and birds 2 to 4 (white band). The band or shadow are 95\% confidence intervals.

Figure 5. Graphical summary of the influence of midazolam-butorphanol-isoflurane on LFP. Although the drugs affected the LFP, the protocol is still feasible for testing responses of the visual pathway with VEPs.

Figure S1. VEP properties when the color stimuli were blocked by a black EVA foam. (A) Averaged LFP traces. (B) Ten examples of raw LFP traces. The triangle indicates the time when the foam was elevated. (C) An averaged spectrogram.

Figure S2. VEP properties after administrating of diazepam for sedation. (A) Averaged LFP

traces. (B) Ten examples of raw LFP traces. The triangle indicates the time of stimulation.

(C) An averaged spectrogram. 
613 Figure S3. All raw LFP traces and ICA pre-processed traces from Bird 5 (control). Blue

614 traces are raw traces (units on Y axes: $\mathrm{mV}$ ). Red traces were pre-processed by ICA (units on

615 Y axes: $\mathrm{mV}$ ) for denoising. The numbers beside each trace represent the orders of the trials.

616 Figure S4. All raw LFP traces and ICA pre-processed traces from Bird 6 (control). Blue

617 traces are raw traces (units on Y axes: $\mathrm{mV}$ ). Red traces were pre-processed by ICA (units on

618 Y axes: $\mathrm{mV}$ ) for denoising. The numbers beside each trace represent the orders of the trials.

619 Figure S5. Averaged LFP traces, spectrogram, and pre-processed spectrogram of Bird 5 and

620 6. (A) and (D) are averaged LFP traces. (B) and (E) are averaged spectrogram of raw LFPs.

621 (C) and (F) are averaged spectrogram of ICA pre-processed LFPs. (A) to (C) are from Bird

6225 else are from Bird 6. 


\section{Figure 1}

Examples of raw LFP traces after watching a light under chemical or physical restraint.

(A) and (B) are graphical abstracts showing the method for recording the evoked potentials. The zebra finches were either restrained by intramuscular administration of midazolam and butorphanol and maintained by inhalation of isoflurane or rolled with a towel. The electrodes were inserted into the left ROT and recorded the LFP while the right eye watched the screen. The screen displayed blacks and greens. (A) is an example of LFP and the evoked potentials of a chemically restrained bird. (B) was obtained with towel restraint. (C) and (D) are ten examples of LFP raw traces. The vertical lines and the arrow heads at zeros are the time points when the black switched to green. 
A

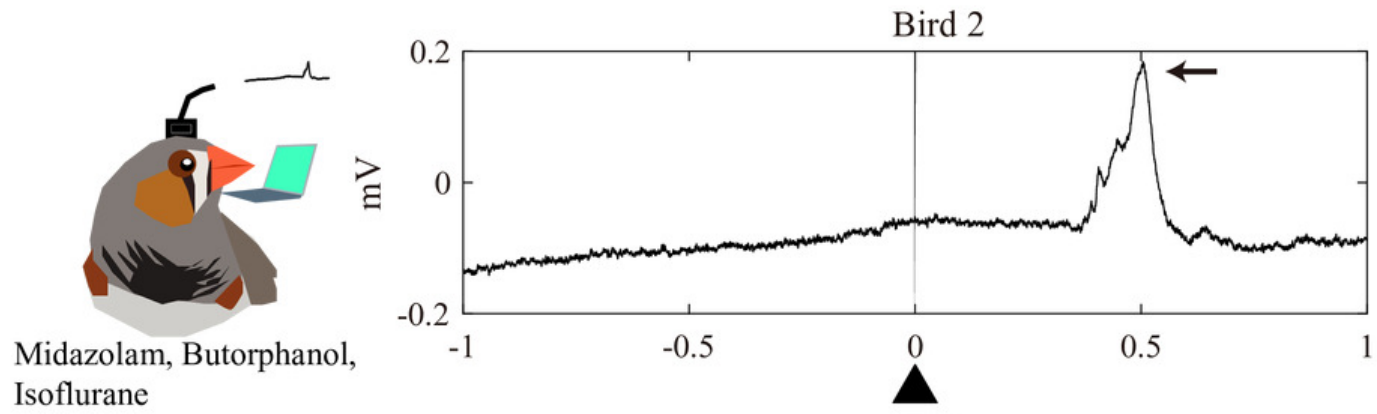

B

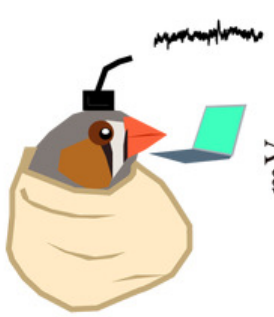

Towel restrained

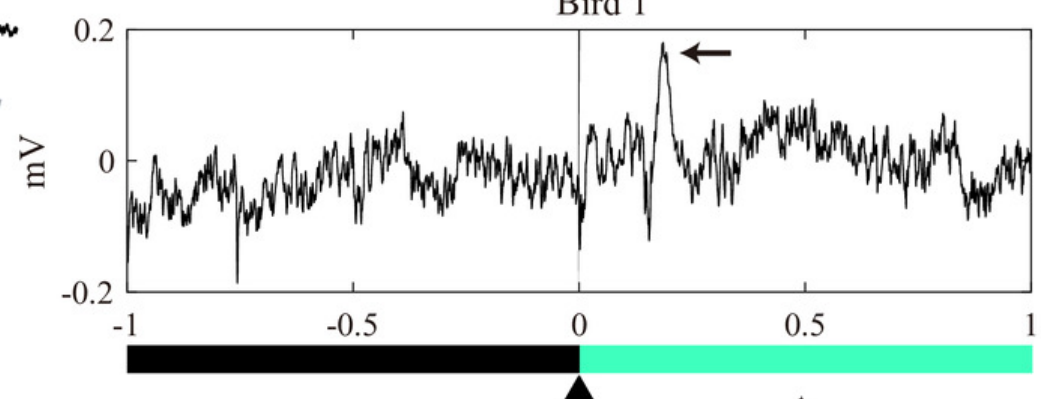

$\mathrm{C}$

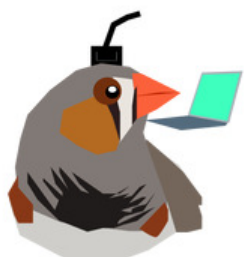

Midazolam, Butorphanol, Isoflurane

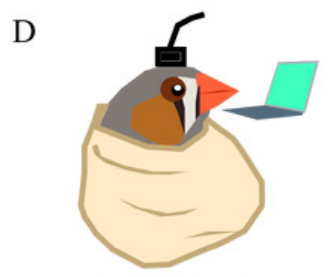

Towel restrained

$0.2 \mathrm{mv}$
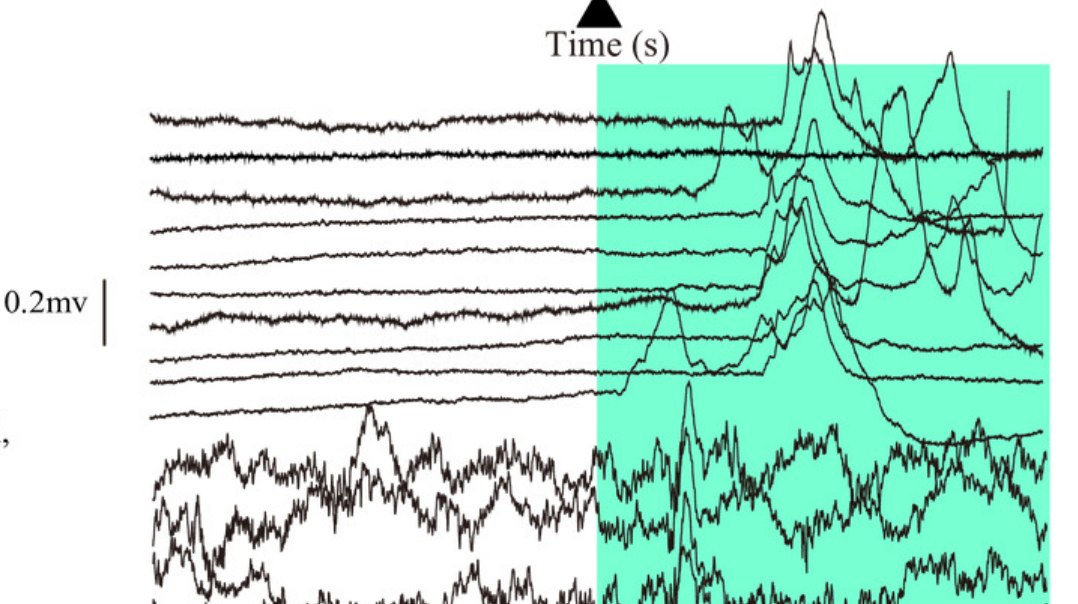

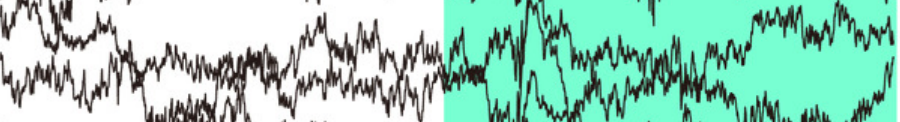
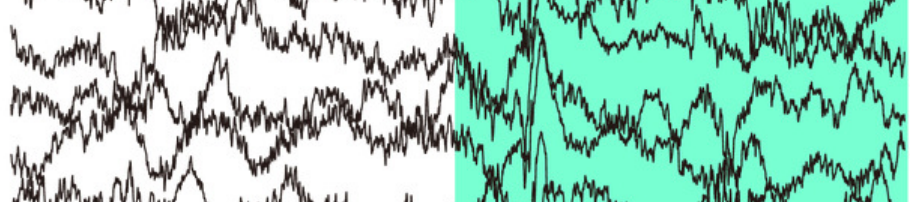

$0.2 \mathrm{mv}$

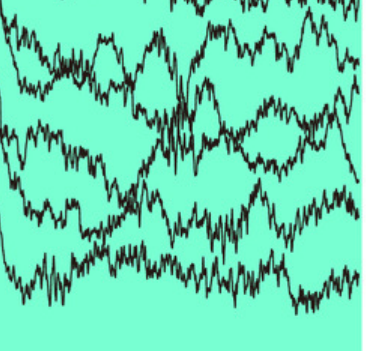

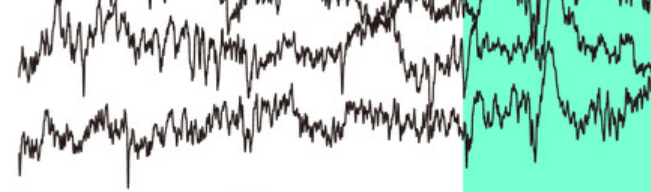

$-1$

Time (s) 
Figure 2

Averaged LFP traces, peak latencies, and peak amplitudes.

(A) to (D) Averaged LFP traces for each subject. The illustration in each figure shows the restraint method. The LFPs were normalized by standard deviation and represented with Zscores. $(E)$ is averaged peak latencies. The Z-scores for peaks are averaged in $(F)$. The lines and shadows and bars and error bars are means \pm SEM. Zero second means the time when the screen changed color from black to green. $*$ denotes the $p$ value $<0.01$. 
A
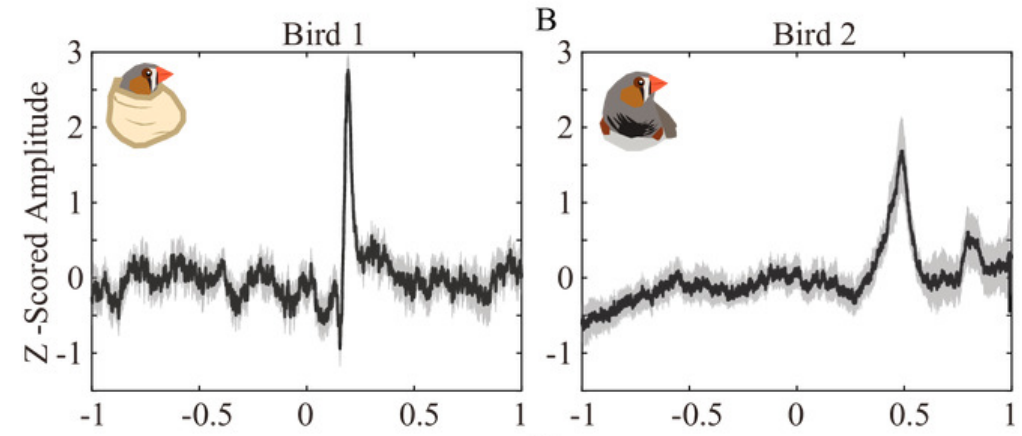

C
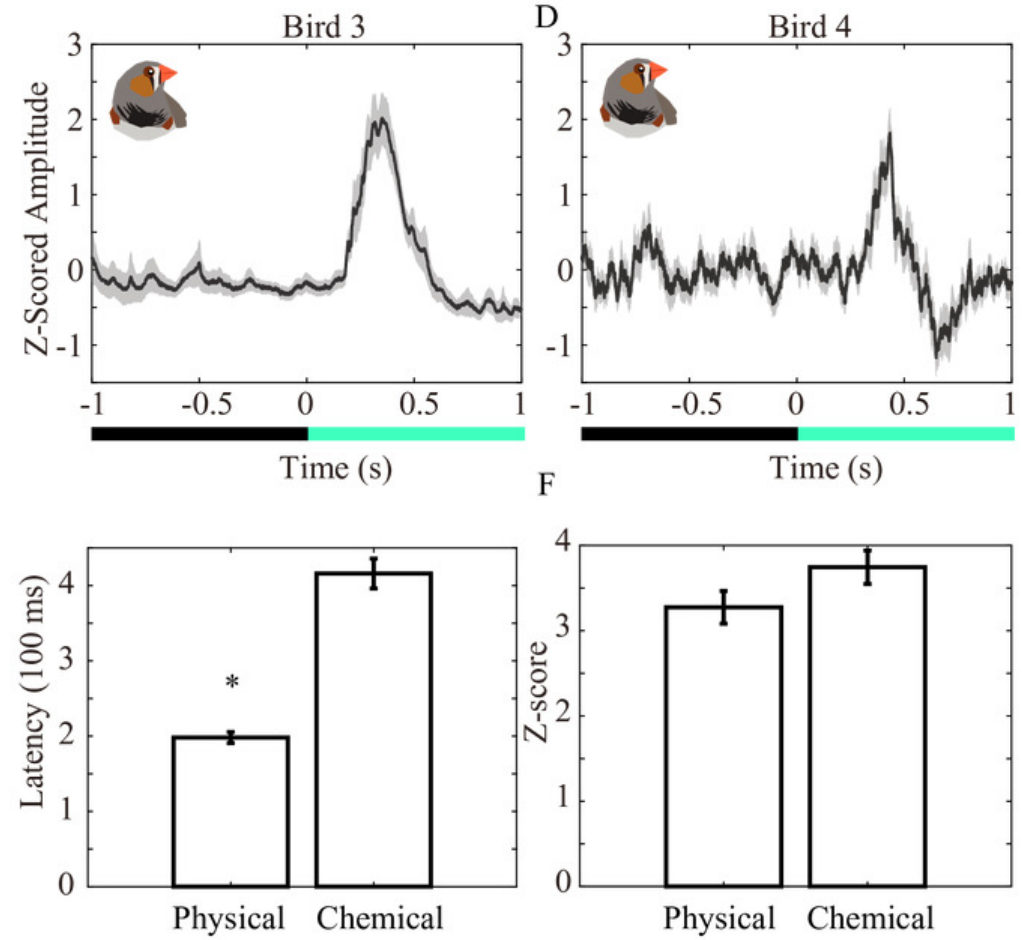

Physical Chemical 


\section{Figure 3}

pre- and poststimulus spectra

(A) to (C) are spectra from one-second LFPs when watching blacks (e.g., -1 to 0 second). (D) to $(F)$ are data from one-second LFP bins after the black changed to green (e.g., 0 to 1 second). The white, red, and blue bands were means $\pm 95 \%$ confidence intervals from Birds 2,3 , and 4 respectively, which were chemically restrained finches. The gray bands are from the physically restrained bird (Bird 1) and illustrate the $95 \%$ confidence intervals. For comparison, the spectra of the physically restrained bird were plotted with the chemically restrained birds. 

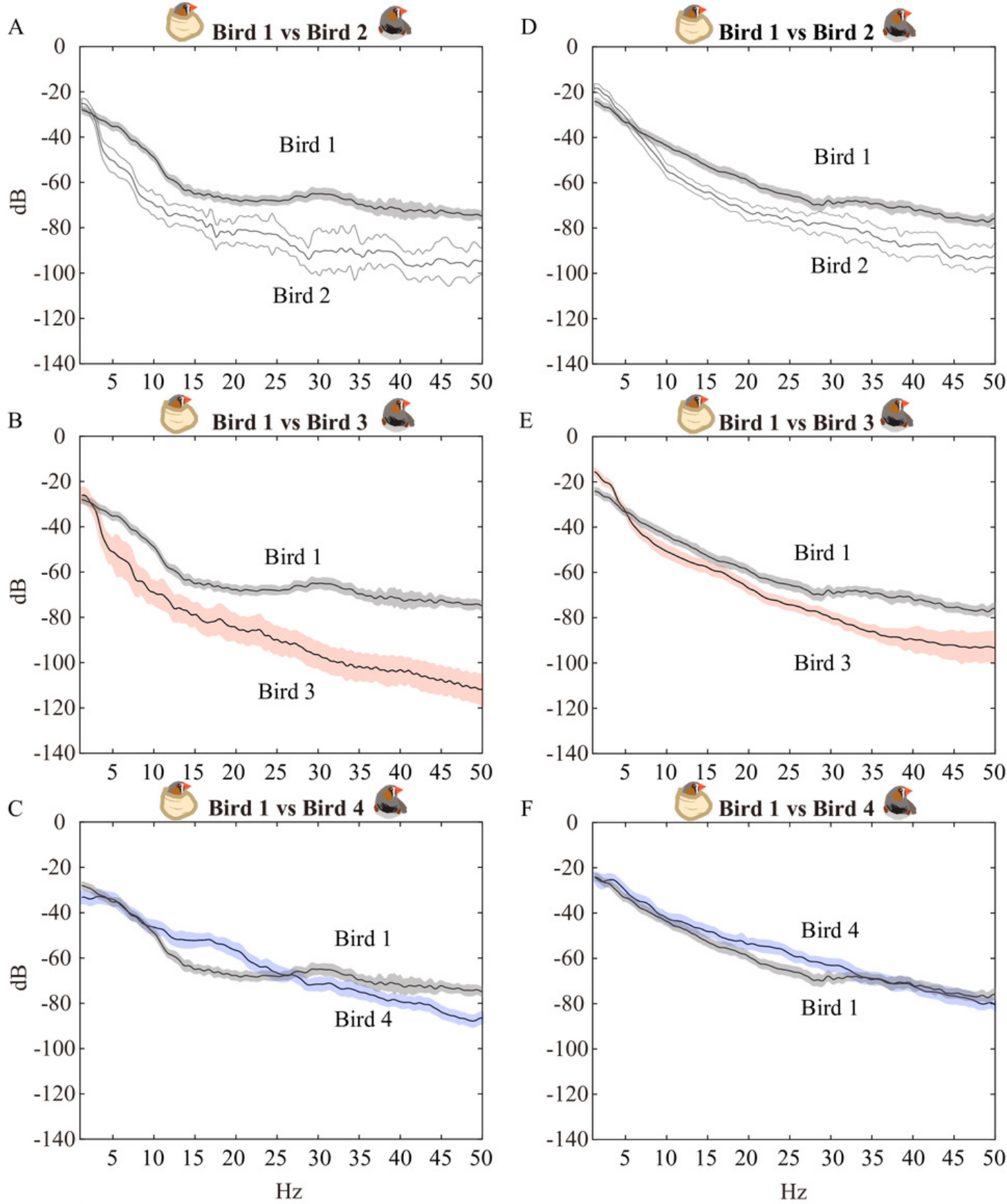


\section{Figure 4}

Averaged spectrogram of each subject and their spectra for VEPs

(A) to (D) Averaged spectrogram of each subject. The warmer color indicates stronger Zscored power at a specific frequency (y-axis) during a specific time (x-axis). The spectra between the dashed lines are displayed in ( $E$ ) and are a comparison between bird 1 (shadow) and birds 2 to 4 (white band). The band or shadow are 95\% confidence intervals. 
A

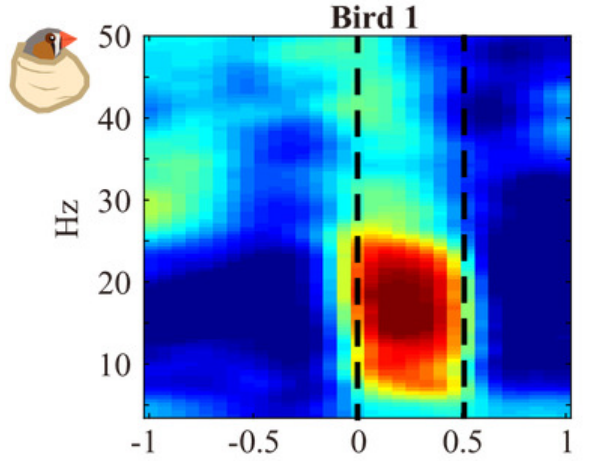

C

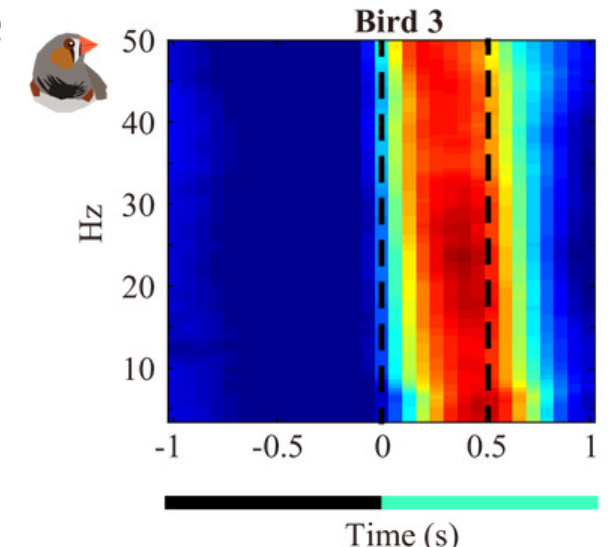

E

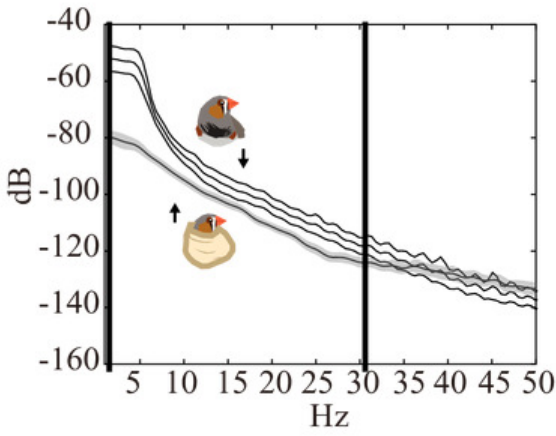

B

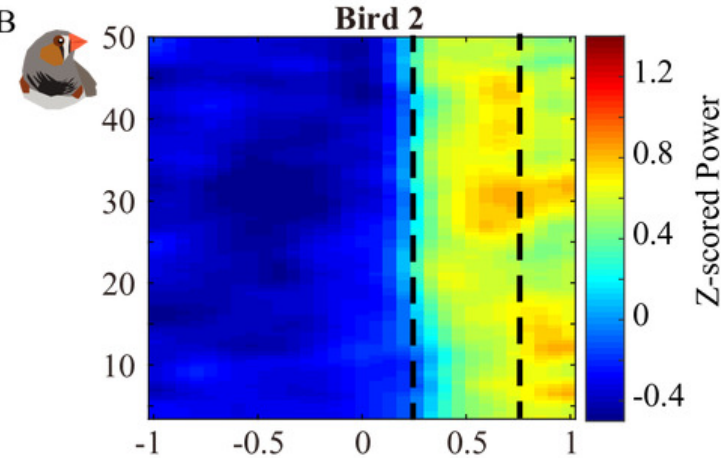

Bird 4

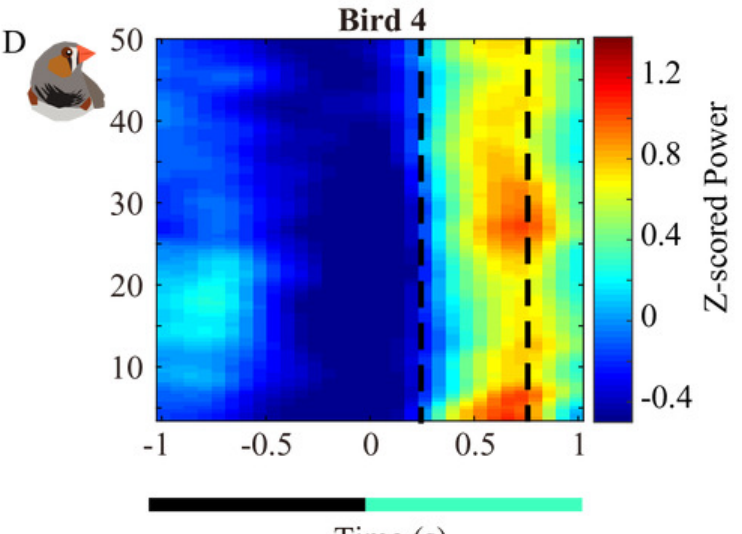

Time (s)

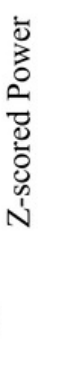

j.




\section{Figure 5}

Graphical summary of the influence of midazolam-butorphanol-isoflurane on LFP.

Although the drugs affected the LFP, the protocol is still feasible for testing responses of the visual pathway with VEPs. 


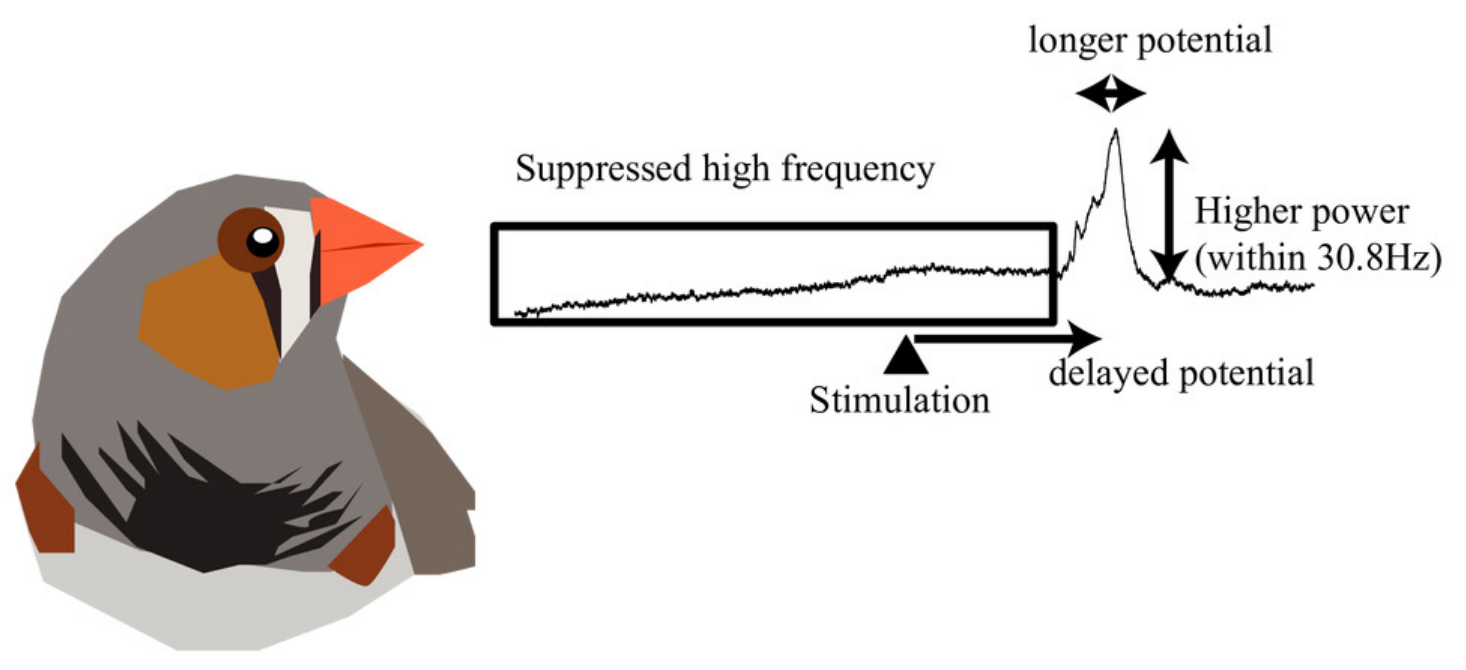

Midazolam and Butorphanol and Isoflurane 